\title{
Clinical Study \\ Quality of Life and Volume Reduction in Women with Secondary Lymphoedema Related to Breast Cancer
}

\author{
Marcus Lanza, ${ }^{1}$ Anke Bergmann, ${ }^{2}$ Maria Giseli da Costa Leite Ferreira, \\ Suzana Sales de Aguiar, ${ }^{2}$ Ricardo de Almeida Dias, ${ }^{2}$ \\ Karen de Souza Abrahão, ${ }^{2}$ Ester M. Paltrinieri, ${ }^{3}$ \\ Ruy G. Martínez Allende, ${ }^{3}$ and Mauro Figueiredo Carvalho de Andrade ${ }^{4}$ \\ ${ }^{1}$ Centro Universitário Augusto Motta, Rio de Janeiro, Brazil \\ ${ }^{2}$ Instituto Nacional de Câncer, Rio de Janeiro, Brazil \\ ${ }^{3}$ Centro Vodder Argentina, Buenos Aires, Argentina \\ ${ }^{4}$ Faculdade de Medicina da Universidade de São Paulo, São Paulo, Brazil
}

Correspondence should be addressed to Anke Bergmann; abergmann@inca.gov.br

Received 17 July 2015; Accepted 24 November 2015

Academic Editor: Debra A. Tonetti

Copyright (c) 2015 Marcus Lanza et al. This is an open access article distributed under the Creative Commons Attribution License, which permits unrestricted use, distribution, and reproduction in any medium, provided the original work is properly cited.

Purpose. To assess the quality of life (QOL) as a predictor of volume reduction in women undergoing complex physical therapy (CPT) for lymphoedema following breast cancer. Methods. Clinical trial in 57 women undergoing CPT. Results. At baseline, in measuring quality of life for the EORTC QLQ-C30 questionnaire subscale of functionality, the worst scores for emotional function (55 points) and better social function ( 89 points) were observed. The symptom scales showed the worst pain averaged (66 points). The overall quality of life showed a low score (40 points). In the BR 23 module, low scores were observed in the field of future perspective (47 points). After treatment of lymphoedema, absolute reduction of excess volume between the upper limbs of $282 \mathrm{~mL}$ was observed, representing a reduction of $15 \%$. No association was observed between the domains of quality of life and response to treatment of lymphoedema. Conclusion. This study included 57 women with advanced and chronic lymphoedema in early treatment with CPT and low scores for quality of life. The lymphoedema therapeutic response was not influenced by the QOL at the beginning of treatment.

\section{Introduction}

Breast cancer has become a major focus of worldwide attention due to the increase in incidence over the past decades. In Brazil, it is an important public health problem, being the most common neoplasm among women and the leading cause of death from cancer in this population group. In 2014, 57,120 new cases were estimated representing a rate of 56.09 cases per 100,000 women [1].

In Brazil, the issue of breast cancer is compounded by the frequency of diagnoses at more advanced stages of the disease. On average, $60 \%$ of cases are diagnosed at stages III and IV, leading to more aggressive and mutilating surgeries, increasing the incidence of complications arising from oncological treatment $[2,3]$.
Lymphoedema is a major complication of treatment for breast cancer, reaching, in our population, about $30 \%$ of women after five years of postoperative follow-up [4].

Several approaches to the treatment of lymphoedema have been reported in the literature $[5,6]$. Among conservative treatments, complex physical therapy (CPT) has emerged as the best approach to control the volume of lymphoedema of the upper limb [7-14]. The first phase of treatment aims for the maximum reduction in limb volume and is part of this treatment: skin care, manual lymph drainage (MLD), exercises, and compression bandaging. Immediately after this phase, the maintenance phase (second phase) starts, consisting of adaptation of compression garments, exercise, and self-massage, with the goal of preserving and optimising the results obtained in the initial phase [10-13]. 
The therapeutic response to any treatment provided depends not only on the physiological actions of the treatment but also on the cultural, social, psychological, and physical conditions of each patient. One of these components, which may influence the therapeutic response, is the quality of life. According to the World Health Organization (WHO) quality of life is defined as "individuals" perception of their position in life in the context of culture, value systems in which they live in relation to their goals, expectations, standards, and concerns [15]. For patients with breast cancer, quality of life assessment will approach the way they experience changes caused by the disease as well as the positive or negative influence that the treatment will have on their lives [16-23].

In this context, this study aims to evaluate QOL as a predictor of treatment response in women undergoing CPT in the treatment of upper limb lymphoedema after axillary lymphadenectomy.

\section{Materials and Methods}

A randomised clinical trial was conducted in women with secondary treatment of breast cancer lymphoedema. The study methodology has been previously detailed [9].

In order to perform the study, 57 women referred for unilateral axillary dissection, with a difference between the upper limbs greater than three centimeters in circumference at one point at least, who were not undergoing chemotherapy or adjuvant radiotherapy and showed no heart disease or systemic decompensated hypertension, were included. Women were excluded if they had surgery less than six months previously, were diagnosed with preoperative lymphoedema, showed signs of inflammation in the swollen limb, had a history of allergic reaction to the material used for compression bandaging, had active locoregional or distant disease, or had been undergoing treatment for lymphoedema with compression bandaging for the last three months.

For the treatment of lymphoedema, all patients underwent complex physical therapy (skin care, compression bandaging, exercises, and home orientations), with or without manual lymphatic drainage.

The main outcome (therapeutic response) was considered to be the percentage reduction in excess limb volume between the beginning and the end of treatment (end of compressive bandaging, occurring in about 4 weeks), calculated by (IV $F V / I V) * 100$, with $I V$ being the initial volume and $F V$ the final volume. The estimated volume of the limb $(V)$ was obtained from the circumference. The measures for each point which were used were $V=h *\left(C^{2}+C * c+c^{2}\right) /(\pi * 12)$, in which $V$ is the volume of the limb segment, $C$ and $c$ are the circumferences at each end, and $h$ is the distance between the circumferences $(C)$.

Descriptive variables were collected, such as age, body mass index (BMI), time to onset postoperative lymphoedema, excess volume in the segment, duration of chronic lymphoedema, and the number of infections in the limb with lymphoedema.
As the main predictor variable, the mean score of quality of life was considered, measured at study entry. Quality of life was assessed by the EORTC QLQ-C30 and BR23, both validated by the Brazilian population [16]. QLQ-C30 is a multidimensional questionnaire designed to assess the psychological and social functioning of patients with cancer. It is made up of 30 questions that assess five functional scales (physical, functional, cognitive, emotional, and social role), three symptom scales (fatigue, pain, nausea, and vomiting), and quality of life scales and overall health. QLQ-BR23 intends to specifically evaluate the effects of treatment in patients with breast cancer. It consists of 23 questions divided into two scales: physical functioning and symptoms. The functional scale is divided into ranges of body image, sexual function, sexual satisfaction, and future perspective. The second level consists of the subscales: adverse effects of systemic therapy, breast symptoms, and arm symptoms. QLQ-C30 and BR23 have a scale ranging from 0 to 100 , in which 0 is the worst health status and 100 the best of health, except for the symptom scales in which higher scores represent more symptoms and worse quality of life. For statistical analysis, the symptom scale was reversed so that higher scores represent better quality of life [15].

The descriptive analysis of the study population was performed by using measures of central tendency and dispersion. To evaluate the changes in excess volume before and after treatment, we performed the Wilcoxon signed-rank test, considered statistically significant at $p<0.05$. Linear regression was performed to evaluate the association between the scores of the domains of quality of life before treatment and the therapeutic response. The statistical package SPSS 20.0 was used for all analyses.

The project was approved by the Ethics Committee in Research of the National Cancer Institute (INCA) under registration number 011/07 and was in accordance with the Helsinki Declaration of 1975, as revised in 2008. All participants signed the informed consent form.

\section{Results}

The study included 57 women. At inclusion in the study, the average age of the study population was 63 years (SD 10.02), mostly overweight and obese. The average time after surgery until the development of lymphoedema was 37 months. At the beginning of treatment, lymphoedema was present for a median of 61 months (5 years). At baseline, the mean excess volume (EV) between the upper limbs was $776.16 \mathrm{~mL}$, corresponding to a percentage excess volume (PEV) of $44.2 \%$ (Table 1).

After treatment, the absolute decrease in statistically significant excess volume between the upper limbs of $282 \mathrm{~mL}$ was observed, representing a reduction of $15 \%(p=0.001)$ (Table 2).

In measuring quality of life for the functional scale of the EORTC QLQ-C30 questionnaire, the worst scores for emotional function (55 points) and better social function (89 points) were observed. In the symptom scales, pain presented the worst average ( 66 points), followed by fatigue 
TABLE 1: Characteristics of patients before the intervention.

\begin{tabular}{lcr}
\hline Variables & Mean (SD) & Median (min.-max.) \\
\hline Age (in years) & $62.87(10.02)$ & $63.95(39-88)$ \\
Time (months) postoperatively for lymphedema & $37.47(55.60)$ & $21.70(1.10-309.47)$ \\
Excess volume (mL) & $776.16(490.19)$ & $652.12(158-2.271)$ \\
Percentage of excess volume & $44.20(26.83)$ & $38.17(7.87-139.91)$ \\
Time (months) with chronic lymphedema & $60.90(62.98)$ & $50.77(0-318)$ \\
Body mass index & $29.75(5.57)$ & $29.40(21-48)$ \\
\hline
\end{tabular}

SD: standard deviation.

(68 points). The overall quality of life showed a low score (40 points). When evaluating the quality of life by BR 23 module, a low score was observed in the field of future perspective (47 points) and arm symptoms (53 points). There was no association between the domains of quality of life and therapeutic response to treatment of lymphedema (Table 3).

\section{Discussion and Conclusion}

Our results showed that the quality of life at the beginning of treatment was not a predictor of therapeutic response in women undergoing CPT in the treatment of upper limb lymphoedema secondary to breast cancer. Different aspects need to be discussed so as to better understand these results, including the sociodemographic and clinical characteristics of women included in this study.

The women studied were from a single public institution, with reference to the treatment of breast cancer (Brazilian National Cancer Institute). Such women are given physical therapy at all stages of cancer treatment with the aim of preventing and minimising complications resulting from breast cancer treatment and its progression [24]. However, even with access to physical therapy, average excess volume of $44 \%$ between the upper limbs at the beginning of treatment was observed. Lymphoedema developed on average 37 months after surgery and was present on average 60 months previously. In another clinical trial with similar methodology conducted with 102 Canadian patients, in most cases (73\%) the average excess volume of the affected limb was between $10 \%$ to $30 \%$ and lymphoedema was present for less than one year in $43 \%$ of patients [11]. In a study conducted with crossover design with 32 patients in the early intervention, $34.4 \%$ of cases were classified as severe lymphoedema ( $>40 \%$ excess volume) and the average duration of lymphoedema was 73 months [25]. Therefore, our population has more advanced and greater chronicity compared to previous lymphoedema studies.

The use of quality of life assessments in breast cancer patients has an important role as a risk for complications, treatment outcome, and prognosis [19, 22, 23, 26]. With regard to quality of life before physiotherapy, when assessed by the EORTC QLQ C-30 instrument, the average for overall quality of life was 40.2. In the functional domain, worse scales for quality of life were in emotional function (mean 54.82) and cognitive function (mean 63.43). On the scale of symptoms, worse quality of life scores were reported for pain (mean 65.7) and fatigue (mean 68.4). When assessing the quality of life by BR 23 module, a low score was observed in the domain of future perspective (47 points) and arm symptoms (53 points).

In a study performed by Gurdal et al. [8], using the EORTC QLQ C-30 instrument and also evaluating before treatment for lymphoedema, the population showed a better mean score of overall quality of life (58.2 and 64.8, depending on the group treatment). In the domain of functionality, the physical and social functions were the most affected and the symptoms of pain and fatigue had the worst score. The difference in the functionality of our patients may reflect cultural issues, especially those related to body image. Physical symptoms associated with lymphoedema include decreased strength and range of motion of the upper limb, fatigue, and pain. These symptoms, associated with the appearance of lymphoedema, can lead to negative self-image, especially in relation to body image, which also affects social and sexual relationships. Emotional well-being is also altered, and these women experience greater stress, anxiety, sadness, anger, frustration, and guilt in relation to their situation [27].

Regarding the response to lymphoedema treatment, several clinical trials and observational studies which have performed CPT report a statistically significant decrease in limb volume before and after treatment [11-14, 28-30]. In our population, the average reduction in excess volume was $282 \mathrm{~mL}$, with $15 \%$ reduction $(p<0.001)$. The best result was observed in a randomised clinical trial of 77 women treated with compression bandaging, exercises, and MLD group. A reduction rate of $36 \%$ and $56 \%$ was reported (according to treatment group) [12]. This difference in treatment response can be explained by the protocols established in each service. In our population, as it is within a public hospital with great demand for lymphoedema treatment, patients were treated twice a week, using compression bandaging throughout the period, which may explain the worse therapeutic response.

In this study, we found no association between quality of life at the beginning of treatment and the therapeutic response of the CPT in any studied domain. Neither did we find in the literature other studies that have evaluated these variables.

This study has some limitations, among which we can highlight the sample size which may have influenced the lack of association between the therapeutic response and the quality of life symptoms subscale in the arm symptoms 


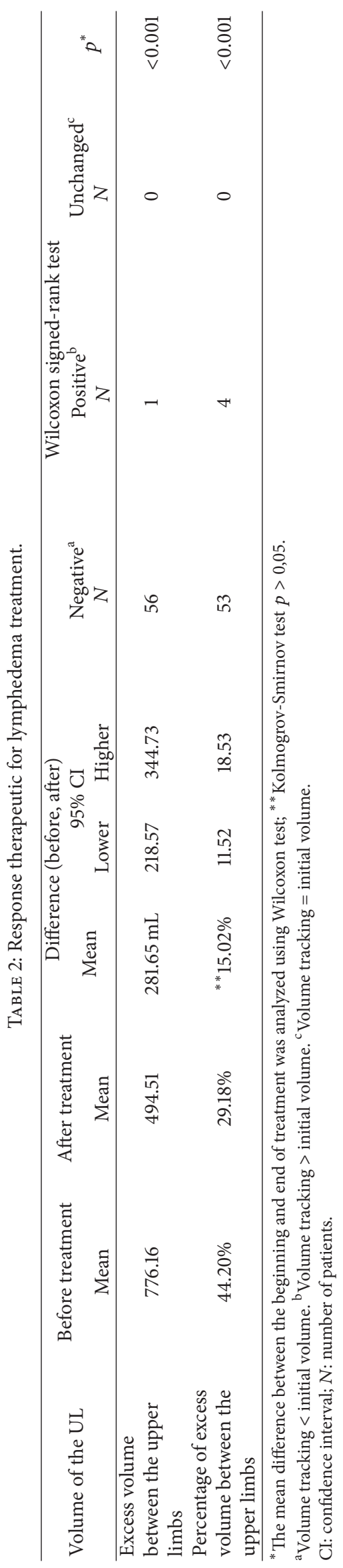


TABLE 3: Linear regression model between the score of quality of life at baseline and percentage reduction of excess volume between the upper limb after treatment.

\begin{tabular}{|c|c|c|c|}
\hline \multirow{2}{*}{ Quality of life } & \multirow{2}{*}{ Mean (SD) } & \multicolumn{2}{|c|}{ Simple linear regression } \\
\hline & & Beta (CI 95\%) & $p$ value \\
\hline \multicolumn{4}{|l|}{ EORTC QLQ C30 } \\
\hline \multicolumn{4}{|l|}{ Functional scale } \\
\hline Physical function & $70.39(21.39)$ & $-0.05(-0.22-0.12)$ & 0.591 \\
\hline Performing roles & $71.16(33.50)$ & $0.03(-0.07-0.14)$ & 0.520 \\
\hline Cognitive function & $63.43(28.95)$ & $0.01(-0.11-0.14)$ & 0.826 \\
\hline Emotional function & $54.82(31.31)$ & $-0.05(-0.17-0.06)$ & 0.388 \\
\hline Social function & $89.42(19.54)$ & $-0.01(-0.20-0.18)$ & 0.917 \\
\hline \multicolumn{4}{|l|}{ Symptoms scale } \\
\hline Dyspnea & $82.70(33.31)$ & $0.05(-0.05-0.16)$ & 0.318 \\
\hline Pain & $65.72(35.90)$ & $0.00(-0.10-0.10)$ & 0.992 \\
\hline Fatigue & $68.37(30.83)$ & $-0.03(-0.15-0.08)$ & 0.563 \\
\hline Insomnia & $76.29(36.29)$ & $-0.04(-0.14-0.05)$ & 0.372 \\
\hline Loss of appetite & $92.30(19.38)$ & $0.01(-0.17-0.20)$ & 0.878 \\
\hline Nausea and vomiting & $92.63(14.54)$ & $0.09(-0.16-0.34)$ & 0.486 \\
\hline Constipation & $83.33(31.30)$ & $-0.04(-0.15-0.08)$ & 0.505 \\
\hline Diarrhea & $94.87(19.10)$ & $-0.02(-0.21-0.17)$ & 0.863 \\
\hline Financial hardship & $76.94(38.20)$ & $0.07(-0.02-0.16)$ & 0.139 \\
\hline Overall quality of life & $40.20(29.18)$ & $0.02(-0.10-0.15)$ & 0.728 \\
\hline \multicolumn{4}{|l|}{$B R 23$} \\
\hline \multicolumn{4}{|l|}{ Functional scale } \\
\hline Body image & $76.37(29.02)$ & $0.01(-0.11-0.14)$ & 0.826 \\
\hline Future perspective & $47.28(40.90)$ & $-0.03(-0.12-0.05)$ & 0.448 \\
\hline Sexual function & $76.06(25.00)$ & $0.00(-0.14-0.15)$ & 0.951 \\
\hline \multicolumn{4}{|l|}{ Symptoms scale } \\
\hline Systemic therapy & $75.24(20.23)$ & $0.08(-0.10-0.25)$ & 0.385 \\
\hline Arm symptoms & $53.34(27.16)$ & $-0.11(-0.24-0.02)$ & 0.099 \\
\hline Breast symptoms & $83.49(21.36)$ & $-0.00(-0.17-0.17)$ & 0.994 \\
\hline
\end{tabular}

subscale $(p=0.099)$. In other scales evaluated, it is unlikely that an increase in the sample size would change the result. As strengths, we highlight the homogeneity of women and little possibility of selection and classification bias, since the data were collected from patients from a single institution in reference to the treatment of breast cancer, which gives adequate internal validity of the study. However, generalization of the results (external validity) should be viewed with caution. Studies in other populations with different treatment and sociodemographic conditions could be conducted to better understand the influence of quality of life as a predictor of therapeutic response of lymphoedema.

In conclusion, this study included 57 women with advanced and chronic lymphoedema in early treatment with CPT. Domain scores of quality of life observed at inclusion of the study had worse scores for emotional function and better scores for social function. In symptom scales, pain and fatigue presented the worst average (68 points). Overall quality of life scores were low. When evaluating the quality of life by BR 23 module, a low score was observed in the field of future perspective and arm symptoms. After treatment, absolute reduction of excess volume between the upper extremities of $282 \mathrm{~mL}$ was observed, representing a reduction of $15 \%$. No association was observed between the domains of quality of life and therapeutic response to treatment of lymphoedema.

\section{Disclosure}

English language editing was carried out by proof-readingservice.com (Reference no. 201410-624816).

\section{Conflict of Interests}

All authors declare that no competing financial interests exist.

\section{Acknowledgment}

The authors acknowledge the Physiotherapy Department of the Hospital's Cancer III/National Cancer Institute. 


\section{References}

[1] Brasil, Ministério da Saúde, Instituto Nacional de Câncer, Estimativa de câncer no Brasil, 2014, http://www2.inca.gov.br.

[2] E. A. N. Fabro, A. Bergmann, B. D. A. e Silva et al., "Postmastectomy pain syndrome: incidence and risks," The Breast, vol. 21, no. 3, pp. 321-325, 2012.

[3] A. Bergmann, V. V. Mendes, R. de Almeida Dias, B. do Amaral E Silva, M. G. da Costa Leite Ferreira, and E. A. N. Fabro, "Incidence and risk factors for axillary web syndrome after breast cancer surgery," Breast Cancer Research and Treatment, vol. 131, no. 3, pp. 987-992, 2012.

[4] J. L. B. Bevilacqua, M. W. Kattan, Y. Changhong et al., "Nomograms for predicting the risk of arm lymphedema after axillary dissection in breast cancer," Annals of Surgical Oncology, vol. 19, no. 8, pp. 2580-2589, 2012.

[5] M. Moattari, B. Jaafari, A. Talei et al., "The effect of combined decongestive therapy and pneumatic compression pump on lymphedema indicators in patients with lymphedema secondary to breast cancer treatment: a randomized clinical control trial," Breast Journal, vol. 19, no. 1, pp. 114-115, 2013.

[6] J. W. Granzow, J. M. Soderberg, and C. Dauphine, "A novel two-stage surgical approach to treat chronic lymphedema," The Breast Journal, vol. 20, no. 4, pp. 420-422, 2014.

[7] N. Devoogdt, M.-R. Christiaens, I. Geraerts et al., "Effect of manual lymph drainage in addition to guidelines and exercise therapy on arm lymphoedema related to breast cancer: randomised controlled trial," British Medical Journal, vol. 343, no. 7824, Article ID d5326, 2011.

[8] S. O. Gurdal, A. Kostanoglu, I. Cavdar et al., "Comparison of intermittent pneumatic compression with manual lymphatic drainage for treatment of breast cancer-related lymphedema," Lymphatic Research and Biology, vol. 10, no. 3, pp. 129-135, 2012.

[9] A. Bergmann, M. G. da Costa Leite Ferreira, S. S. de Aguiar et al., "Physiotherapy in upper limb lymphedema after breast cancer treatment: a randomized study," Lymphology, vol. 47, no. 2, pp. 82-91, 2014.

[10] International Society of Lymphology, "Consensus Document of The International Society of Lymphology. The diagnosis and treatment of peripheral lymphedema," Lymphology, vol. 46, no. 1, pp. 1-11, 2013.

[11] I. S. Dayes, T. J. Whelan, J. A. Julian et al., "Randomized trial of decongestive lymphatic therapy for the treatment of lymphedema in women with breast cancer," Journal of Clinical Oncology, vol. 31, no. 30, pp. 3758-3763, 2013.

[12] K. Didem, Y. S. Ufuk, S. Serdar, and A. Zümre, "The comparison of two different physiotherapy methods in treatment of lymphedema after breast surgery," Breast Cancer Research and Treatment, vol. 93, no. 1, pp. 49-54, 2005.

[13] M. L. McNeely, D. J. Magee, A. W. Lees, K. M. Bagnall, M. Haykowsky, and J. Hanson, "The addition of manual lymph drainage to compression therapy for breast cancer related lymphedema: a randomized controlled trial," Breast Cancer Research and Treatment, vol. 86, no. 2, pp. 95-106, 2004.

[14] R. Koul, T. Dufan, C. Russell et al., "Efficacy of complete decongestive therapy and manual lymphatic drainage on treatmentrelated lymphedema in breast cancer," International Journal of Radiation Oncology Biology Physics, vol. 67, no. 3, pp. 841-846, 2007.

[15] The World Health Organization Quality of Life (WHOQOL), 2013, http://www.who.int/mental_health/publications/whoqol/ en/.
[16] M. Tsuchiya, "Patient education, upper-limb symptom perception, and quality of life among Japanese breast cancer survivors," Quality of Life Research, vol. 23, no. 8, pp. 2327-2332, 2014.

[17] K. T. Ashing-Giwa and J.-W. Lim, "Predicting physical quality of life among a multiethnic sample of breast cancer survivors," Quality of Life Research, vol. 19, no. 6, pp. 789-802, 2010.

[18] T. S. Dabakuyo, F. Guillemin, T. Conroy et al., "Response shift effects on measuring post-operative quality of life among breast cancer patients: a multicenter cohort study," Quality of Life Research, vol. 22, no. 1, pp. 1-11, 2013.

[19] S. S. De Aguiar, A. Bergmann, and I. E. Mattos, "Quality of life as a predictor of overall survival after breast cancer treatment," Quality of Life Research, vol. 23, no. 2, pp. 627-637, 2014.

[20] B. L. Den Oudsten, J. De Vries, A. F. W. Van der Steeg, J. A. Roukema, and G. L. Van Heck, "Determinants of overall quality of life in women over the first year after surgery for early stage breast cancer," Quality of Life Research, vol. 18, no. 10, pp. 13211329, 2009.

[21] E. Farin and M. Nagl, "The patient-physician relationship in patients with breast cancer: influence on changes in quality of life after rehabilitation," Quality of Life Research, vol. 22, no. 2, pp. 283-294, 2013.

[22] P. A. Ganz, "Quality of life assessment in breast cancer: when does it add prognostic value for survival?" Breast Journal, vol. 17, no. 6, pp. 569-570, 2011.

[23] C. M. Chen, S. J. Cano, A. F. Klassen et al., "Measuring quality of life in oncologic breast surgery: a systematic review of patientreported outcome measures," Breast Journal, vol. 16, no. 6, pp. 587-597, 2010.

[24] A. Bergmann, M. J. P. Ribeiro, E. Pedrosa, E. A. Nogueira, and A. C. G. Oliveira, "Physical therapy in breast cancer: clinical protocol at the cancer hospital III/INCA," Revista Brasileira de Cancerologia, vol. 52, pp. 97-109, 2006.

[25] R. Belmonte, M. Tejero, M. Ferrer et al., "Efficacy of lowfrequency low-intensity electrotherapy in the treatment of breast cancer-related lymphoedema: a cross-over randomized trial," Clinical Rehabilitation, vol. 26, no. 7, pp. 607-618, 2012.

[26] E. D. Staren, D. Gupta, and D. P. Braun, "The prognostic role of quality of life assessment in breast cancer," The Breast Journal, vol. 17, no. 6, pp. 571-578, 2011.

[27] N. R. Taghian, C. L. Miller, L. S. Jammallo, J. O’Toole, and M. N. Skolny, "Lymphedema following breast cancer treatment and impact on quality of life: a review," Critical Reviews in Oncology/Hematology, vol. 92, no. 3, pp. 227-234, 2014.

[28] J. B. Hamner and M. D. Fleming, "Lymphedema therapy reduces the volume of edema and pain in patients with breast cancer," Annals of Surgical Oncology, vol. 14, no. 6, pp. 19041908, 2007.

[29] D. Karadibak, T. Yavuzsen, and S. Saydam, "Prospective trial of intensive decongestive physiotherapy for upper extremity lymphedema," Journal of Surgical Oncology, vol. 97, no. 7, pp. 572-577, 2008.

[30] S. Vignes, R. Porcher, M. Arrault, and A. Dupuy, "Factors influencing breast cancer-related lymphedema volume after intensive decongestive physiotherapy," Supportive Care in Cancer, vol. 19, no. 7, pp. 935-940, 2011. 


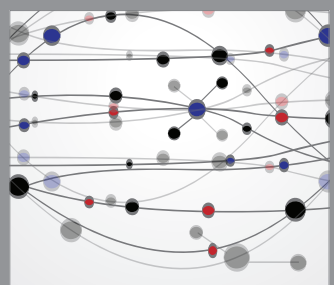

The Scientific World Journal
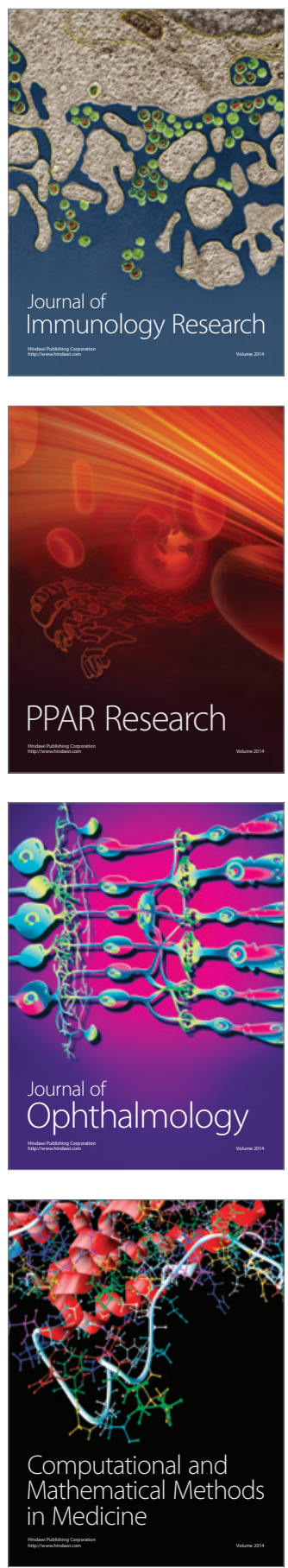

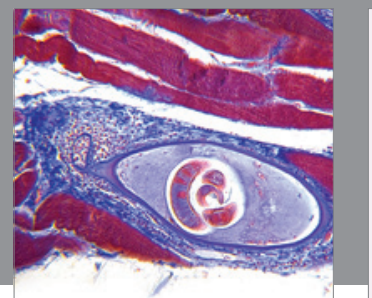

Gastroenterology

Research and Practice
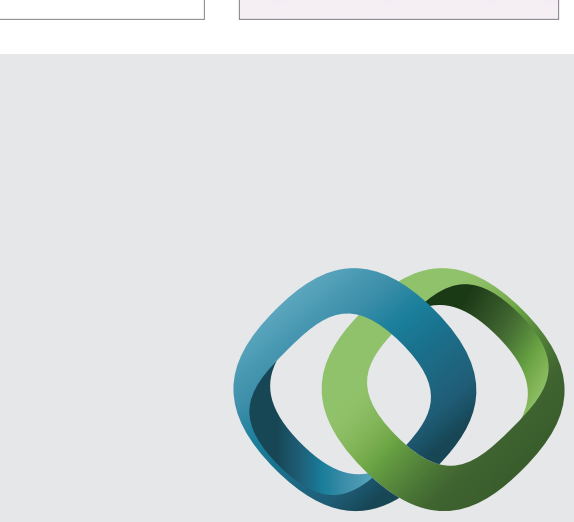

\section{Hindawi}

Submit your manuscripts at

http://www.hindawi.com
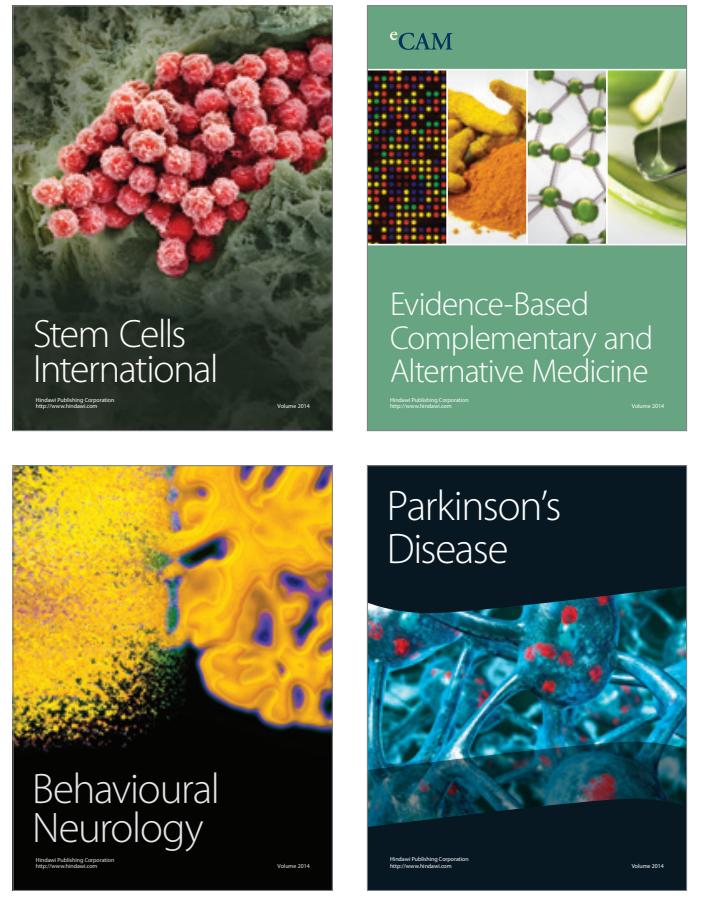
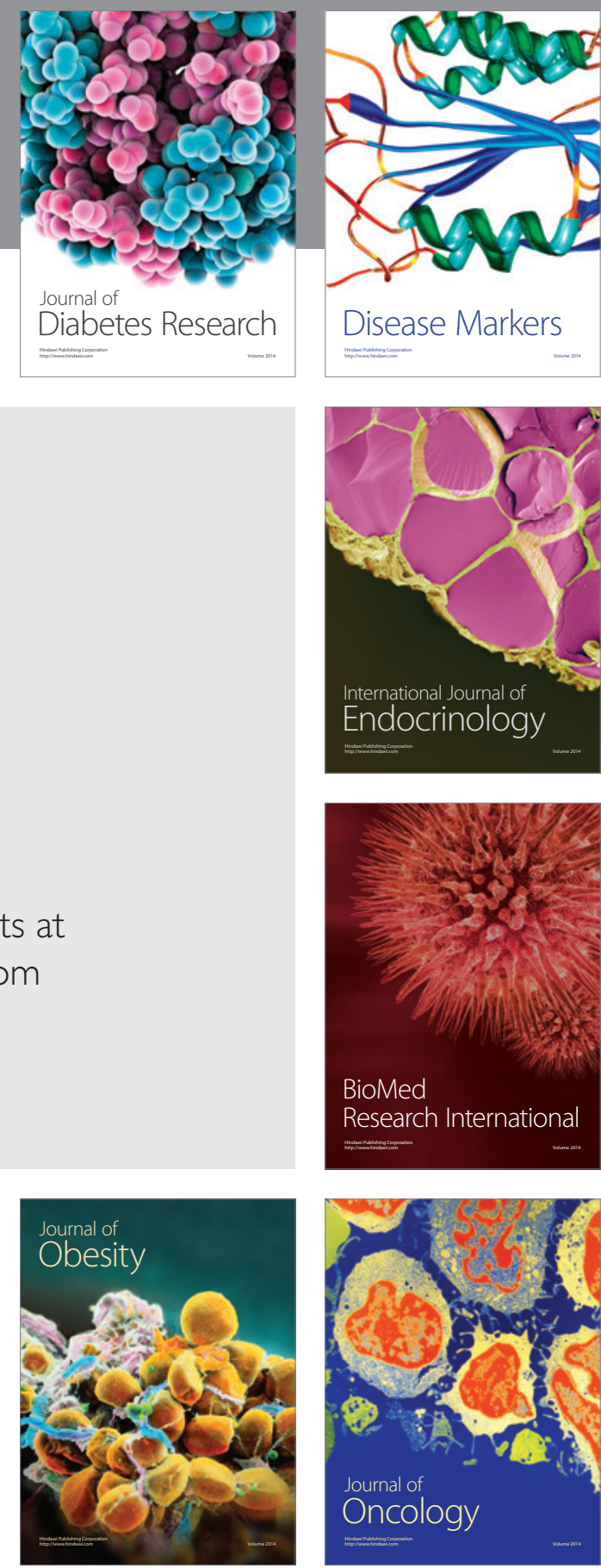

Disease Markers
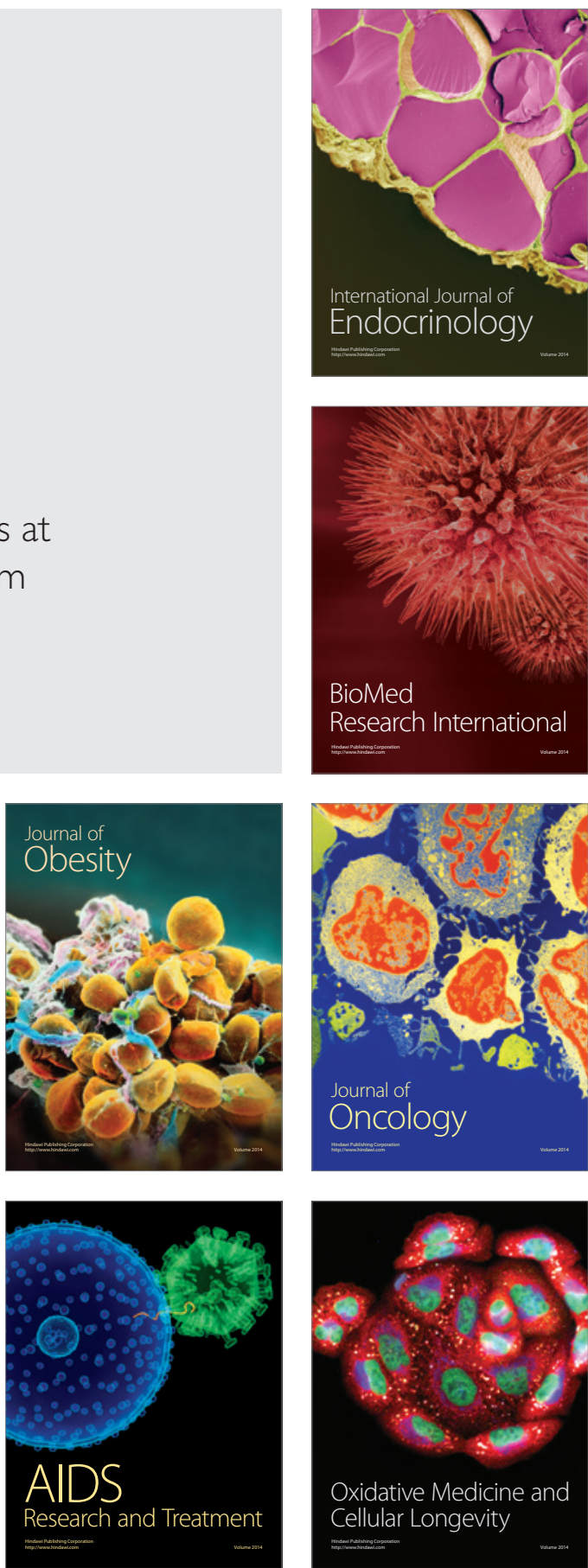